\title{
A 3-Dimensional Analysis of Electrooptic Modulators by the Method of Lines
}

\author{
Shyh-Jong Chung \\ Institute of Communication Eng., National Chiao Tung University \\ Hsinchu, Taiwan, R.O.C.
}

\begin{abstract}
A 3-dimensional analysis using the method of lines is developed to study the electrostatic field within an electrooptic switch/modulator. The effect of the ends of the electrodes is investigated by considering the distributions of the electric field along the transverse and longitudinal directions. Also, the capacitance related to the abrupt ends is calculated for several parameters of the structure.
\end{abstract}

\section{INTRODUCTION}

Electrooptic (EO) modulators are widely used in integrated optical circuits or electrooptic integrated circuits as a connection between optical and electrical signals [1][3]. They are also used in microwave circuits [4]. In these devices, a controlling electric field is applied to the substrate to perturb the permittivity of the guiding medium through the electrooptic (Pockel) effect, achieving modulation of the guided optical wave. The depth of the modulation relies both on the overlap integral factor [5] and the length of the modulator.

Many authors have paid their attentions to the analysis of the field distribution near the electrodes of the EO modulators, with approaches such as comformal mapping [6]-[8], the method of lines [9], finite element method [10][11], and point-matching method [12][13]. For simplicity the end effects of electrodes are ignored so that a twodimensional problem is tackled in their analysis. However, for a modulator operated under several $\mathrm{GHz}$, the electrode lengths are much smaller than the wavelength. It seems that a three-dimensional analysis is needed to get more complete informations about the electric fields in the modulators.

In this study the author implements a static analysis of EO modulators using a 3-dimensional method of lines, with special emphasis on the end effect of the electrodes. Section II describes the method of the analysis. Since the

Manuscript received May 28,1992. This work was supported by the National Science Council of the Republic of China under Grant NSC 81-0404-E-009-529. method of lines is well-documented in [14], only a brief summary is given. Section III discusses some numerical results followed by conclusions in section IV.

\section{ANALYSIS}

The structure of the EO modulator for analysis is shown in Fig. 1, where two infinitely thin electrodes are placed on a buffer layer $\mathrm{SiO}_{2}$ which in turn adheres to an anisotropic material $\mathrm{LiNbO}_{3}$. Since the change in dielectric constant of $\mathrm{LiNbO}_{3}$ due to $\mathrm{Ti}$ diffusion used in fabricating the waveguides is rather small, its effect is neglected in the analysis. The whole structure is enclosed by perfect electric conductors (PEC) except at the side of $z=-L$ where a perfect magnetic conductor (PMC) is introduced either due to the symmetry of the geometry or due to the invariances (in the $z$ direction) of the field and charge distributions at $z<-L$. By noting that the dimensions of the structure are very small compared to the operating wavelength, an electrostatic approach is used in the following analysis.

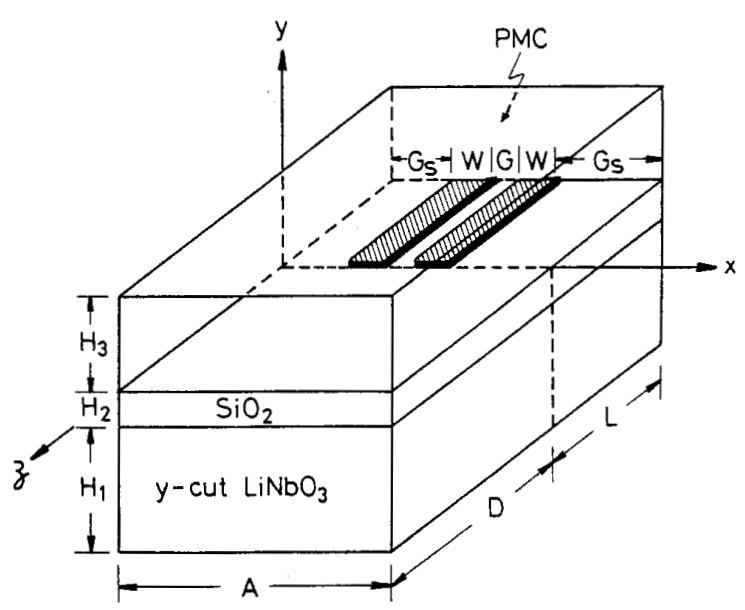

Fig.1. Structure for analysis. 
Let $\mathbf{E}(x, y, z)$ and $\phi(x, y, z)$ be the electric field and potential distributions, respectively. Then

$$
\mathbf{E}=-\nabla \phi \quad,
$$

and $\phi(x, y, z)$, in each layer, is a solution of the Laplace equation :

$$
\epsilon_{r x} \frac{\partial^{2} \phi}{\partial x^{2}}+\epsilon_{r y} \frac{\partial^{2} \phi}{\partial y^{2}}+\epsilon_{r z} \frac{\partial^{2} \phi}{\partial z^{2}}=0
$$

Here the relative permittivity tensor of the layer of $\mathrm{LiNbO}_{3}$ is assumed to be diagonal. Note that in the regions of air and $\mathrm{SiO}_{2}$, the materials are isotropic so that $\epsilon_{r x}=\epsilon_{r y}=\epsilon_{r z}$.

The boundary conditions (B.C.) subjected to (2) are :

1) At the enclosure walls :

$$
\begin{array}{ll}
\phi=0 & , \text { for PEC } \\
\frac{\partial \phi}{\partial z}=0 & , \text { for PMC }
\end{array}
$$

2) At the interface between $\mathrm{SiO}_{2}$ and $\mathrm{LiNbO}_{3}$ :

$$
\phi \text { and } \epsilon_{r y} \frac{\partial \phi}{\partial y} \text { both be continuous. }
$$

3) At the interface between $\mathrm{SiO}_{2}$ and air $(y=0)$ :

$$
\begin{gathered}
\phi \text { be continuous and equal to the potential } V_{k} \\
\text { applied to the } \mathrm{k} \text {-th electrode, } \mathrm{k}=1,2 ; \\
\epsilon_{r y} \frac{\partial \phi}{\partial y}\left(y=0^{-}\right)-\epsilon_{r y} \frac{\partial \phi}{\partial y}\left(y=0^{+}\right)=\frac{\rho(x, z)}{\epsilon_{o}} .
\end{gathered}
$$

Where $\rho(x, z)$ represents the charge distribution on the plane of $y=0$, which is zero outside the electrode region, and $\epsilon_{o}$ is the permittivity of the free space. The superscript " - "and "+ " mean the lower and upper sides of the interface, respectively.

Equation (2) is solved by the method of lines [14]. First by further considering the symmetry of the structure in the $x$ direction (let $V_{1}=-V_{2}=V$ ), a PEC wall is placed at the plane of $x=A / 2$ to simply the analysis. In the implementation of the method of lines, a finite difference procedure is used in the $x$ and $z$ directions to handle the derivatives in (2). Fig. 2 shows the top view of the reduced structure and the locations of the so-called "e-lines" and "h-lines". The potential and its second derivative are evaluated on the e-lines and the first derivative of the potential is evaluated on the h-lines. To reduce the number of variables, the lines near the end of the electrode are denser than those elsewhere by recognizing that the potential has the most variety near the end. Note that e-lines (h-lines) should be put along the PEC (PMC) and the values on these lines are set to be zeros by B.C. 1 .

Following the procedures decribed in [14], a set of coupled differential equation is derived after discretizing (2)

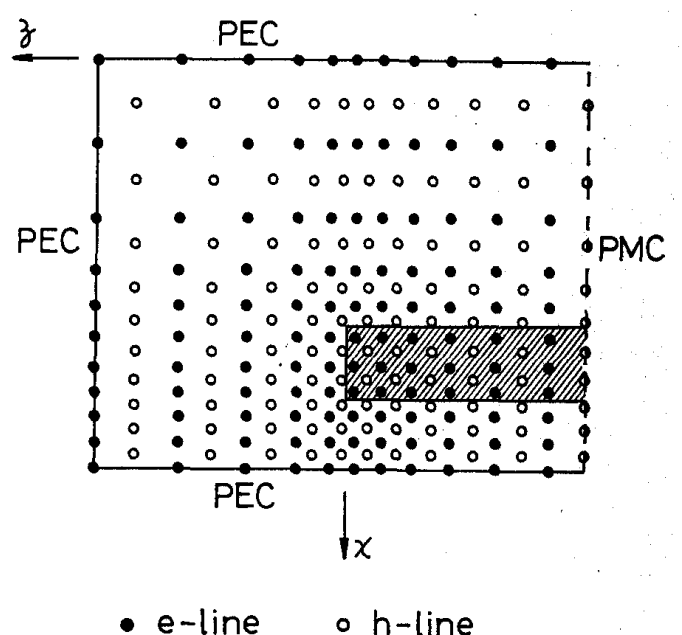

Fig.2. Top view of the reduced structure showing the locations of e-lines and $\mathrm{h}$-lines.

in the $x$ and $z$ directions. By introducing the diagonal eigenvalue matrix $\hat{\boldsymbol{\Lambda}}_{x}^{2}\left(\hat{\boldsymbol{\Lambda}}_{z}^{2}\right)$ and the orthogonal eigenvector matrix $\hat{\mathbf{T}}$ belonging to the difference operator $\hat{\mathbf{P}}_{x}\left(\hat{\mathbf{P}}_{z}\right)$, these equations can be decoupled to

$$
\left(\frac{d^{2}}{d y^{2}} \hat{\mathbf{I}}-\frac{\epsilon_{r x} \hat{\Lambda}_{x}^{2}}{\epsilon_{r y} h_{x}^{2}}-\frac{\epsilon_{r z} \hat{\mathbf{\Lambda}}_{z}^{2}}{\epsilon_{r y} h_{z}^{2}}\right) \overline{\mathbf{\Phi}}=0
$$

In (3), $\hat{\mathbf{I}}$ is the unit matrix, $h_{x}\left(h_{z}\right)$ is the average distance between two adjacent e-lines in the $x(z)$ direction, and $\overline{\mathbf{\Phi}}$ is the potential distribution, which is given by

$$
\overline{\mathbf{\Phi}}=\hat{\mathbf{T}}^{t} \boldsymbol{\Phi}
$$

with superscript " $t$ " denoting the matrix transpose and $\Phi$ representing the potential vector calculated on the elines.

The general solution for the $\mathrm{i}$-th component of $\overline{\boldsymbol{\Phi}}$ can be found as

$$
\bar{\Phi}_{i}=A_{i} \cosh \left(k_{y i} y\right)+B_{i} \sinh \left(k_{y i} y\right)
$$

with

$$
k_{y i}^{2}=\frac{\epsilon_{r x} \hat{\Lambda}_{x i}^{2}}{\epsilon_{r y} h_{x}^{2}}+\frac{\epsilon_{r z} \hat{\Lambda}_{z i}^{2}}{\epsilon_{r y} h_{z}^{2}} .
$$

By imposing B.C. 2) and 3) on (5), an inhomogeneous matrix equation is obtained

$$
\hat{\mathbf{Z}} \bar{\rho}=\overline{\mathbf{\Phi}}
$$

where $\bar{\rho}$ represents the transformed charge distribution on the interface of $y=0$. After transforming back to the spatial domain, and recognizing that charges exist only 
on the electrode and that the electrode potential is equal to $V$, a reduced matrix can be obtained

$$
\mathbf{Z}^{r} \rho_{e l}=\mathbf{\Phi}_{e l}
$$

where the subscript " $e l$ " denotes that the vectors contain only the elements on the e-lines crossing the electrode. $\boldsymbol{\Phi}_{e l}$ is a constant vector whose elements are equal to $V$.

Eq. (8) is inverted to get the charge distribution on the electrode, the potential and electric field are then obtained from (7), (5), (4), and (1).

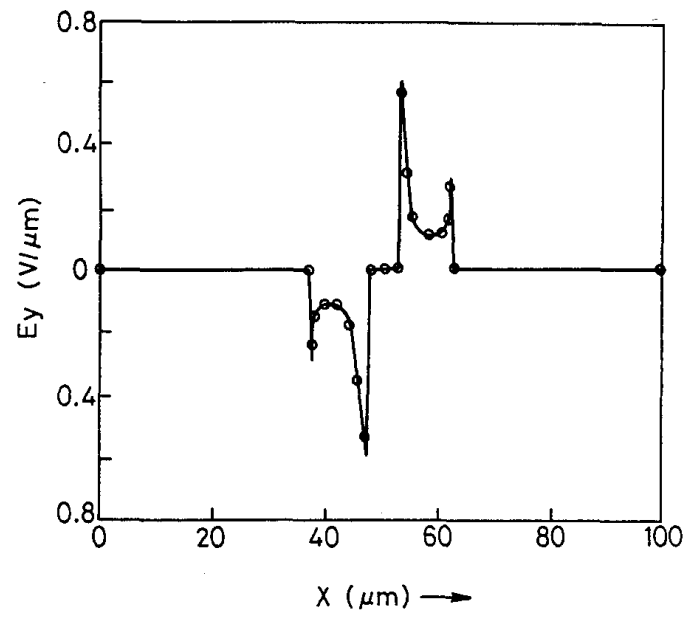

(a)

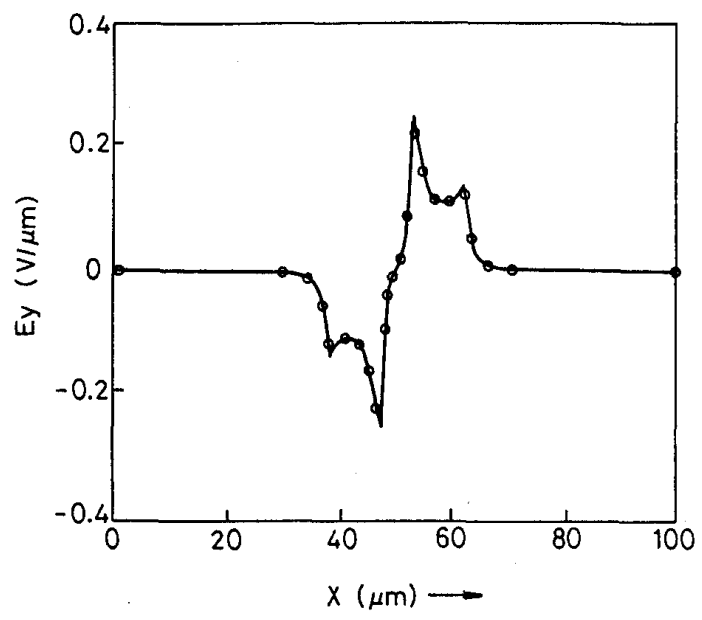

(b)

Fig.3. Distributions of the normal electric fields $\left(E_{y}\right)$ along the lines (a) $(y=0, z=-80 \mu m)$ and (b) $(y=-0.75 \mu m, z=-80 \mu m)$. The bottom $\mathrm{LiNbO}_{3}$ layer is replaced by $\mathrm{SiO}_{2}\left(\epsilon_{r x}=\epsilon_{r y}=\epsilon_{r z}=3.8\right)$. _- present analysis; 000 results from [12].

\section{RESULTS}

As a check of the validity of the computer program, Fig. $3(\mathrm{a})$ and (b) show the normal electric fields $\left(E_{y}\right)$ of the present approach, which are scanned at $z=-80 \mu \mathrm{m}$, compared with those obtained by the two-dimensional point matching method [12]. In this illustration the bottom $\mathrm{LiNbO}_{3}$ layer is replaced by $\mathrm{SiO}_{2}$ for the purpose of comparison. The electric fields are expected to reach the stable values at $z=-80 \mu m$, namely, the results should be equal to those calculated from two-dimensional approach. The close agreements of the curves in Fig. 3 verify the validity of the present analysis.

In the following discussions, the relative permittivity for the $\mathrm{y}$-cut $\mathrm{LiNbO}_{3}$ is set to be $\left(\epsilon_{r x}=\epsilon_{r z}=43, \epsilon_{r y}=28\right)$, and that for $\mathrm{SiO}_{2}$ is $\left(\epsilon_{r x}=\epsilon_{r y}=\epsilon_{r z}=3.8\right)$. The thicknesses of the layers of $\mathrm{LiNbO}_{3}\left(H_{1}\right)$ and air $\left(H_{3}\right)$ are both chosen to be $1 \mathrm{~mm}$. The width $(W)$ and the imposed potential $(V)$ for the electrode are $10 \mu m$ and $1 \mathrm{~V}$, respectively. The length parameters $D, L$, and $G s$ defined in Fig. 1 are 100,100 , and $37.5 \mu m$, respectively. Under the selections of these length parameters, the influence of the side walls (PEC, PMC) on the field behaviors can be neglected. In the discretization, 16 (15) equally spaced e- (h-) lines are located in the $\mathrm{x}$ direction acrossing the electrode range $(G s<x<G s+W)$. Outside this range, the distance between two adjacent e-line and h-line increases geometrically with a ratio of 1.1 . The same ratio is used in the $z$ direction to space the e-lines and h-lines away from the electrode end. Under this discretization, the CPU time for obtaining numerical data from a given structure is about 3 minutes in the VAX- 8800 computer.

Fig. 4 shows the normal electric fields $\left(E_{y}\right)$ at the plane of $y=-H_{2}^{-}$for $z=+20,0,-20$, and $-80 \mu m$. The field at the position $20 \mu \mathrm{m}$-ahead-of the electrode end $(z=+20 \mu m)$ is negligible. Note that the shapes of the three curves for $z=0,-20$, and $-80 \mu m$ are similar, and that the field behavior at $z=-20 \mu m$ is the same as that at $z=-80 \mu m$, which means that the end effect can be neglected as the observation point is $20 \mu m$ away from the end.

Fig. 5 illustrates the distributions of $E_{y}$ in the $z$ direction near the inner edge of one of the electrodes $(x=$ $G s+W-0.16 \mu m)$, calculated at the plane of $y=-H_{2}^{-}$. The field is almost zero outside the electrode. For the case of no buffer layer $\left(\mathrm{H}_{2}=0 \mu \mathrm{m}\right)$, the field approaches infinity when the observation point moves along the electrode and toward its end, which is a consequence of the edge condition.

The capacitance, $\Delta C$, associated with the abrupt stop of the electrodes can be calculated from the difference between the total charge, $Q_{t o t}$, and the charge per unit length of infinitely long electrodes, $Q_{a v e}$, as

$$
\Delta C=\left(Q_{\text {tot }}-L Q_{\text {ave }}\right) / \Delta V,
$$




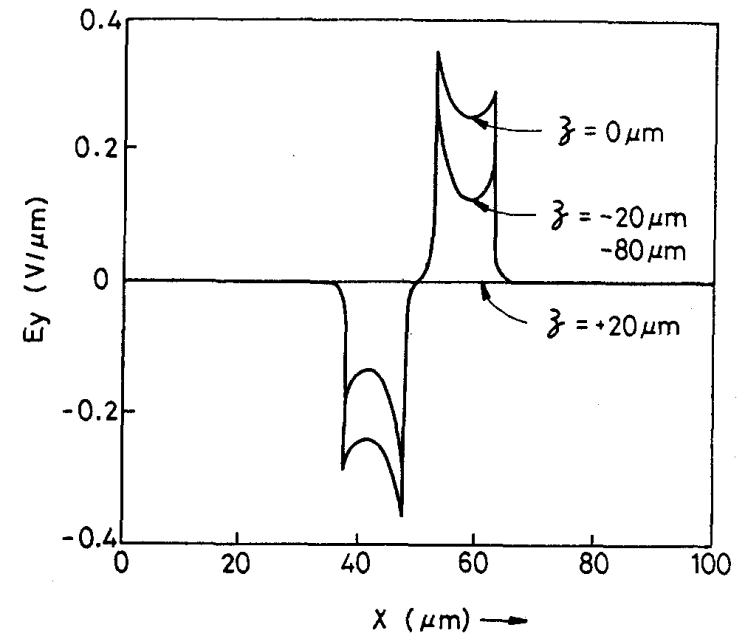

Fig.4. Normal electric fields $\left(E_{y}\right)$ at the plane of $y=-H_{2}^{-}$for $z=+20,0,-20$, and $-80 \mu \mathrm{m} . H_{2}=0.2 \mu \mathrm{m}$.

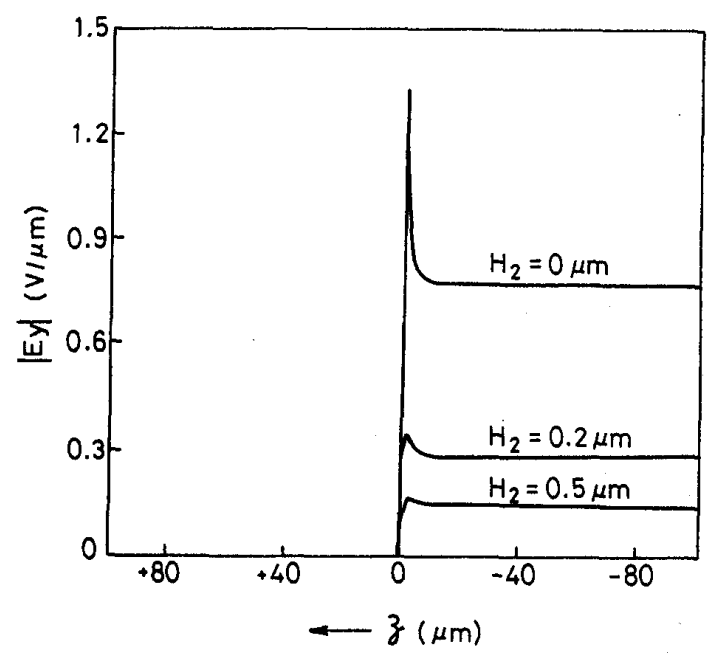

Fig.5. Distributions of the normal electric field $\left(E_{y}\right)$ along the line $\left(x=G s+W-0.16 \mu m, y=-H_{2}^{-}\right)$for $H_{2}=0,0.2$, and $0.5 \mu m$.

where $\Delta V$ is the potential difference of the two electrodes.

Fig. 6 shows the capacitance $\Delta C$ as a function of the gap width $G$. For a given width, the capacitance increases as the buffer layer gets thinner. This is because, when the thickness of the buffer decreases, more electric field concentrates at $\mathrm{LiNbO}_{3}$ which has the higher permittivity than the buffer $\mathrm{SiO}_{2}$. Note that as the gap width increases, although the capacitance per unit length of the uniform electrodes decreases, $\Delta C$ increases. This implies that the end effect of the electrodes gets stronger as the gap width increases.

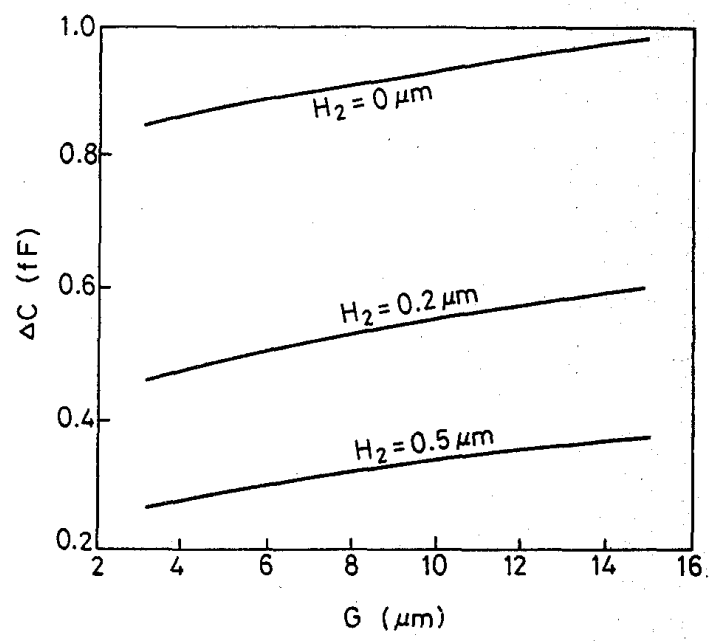

Fig.6. Capacitance $(\Delta C)$ as a function of the center gap width.

\section{CONCLUSIONS}

The author has developed a Fortran program using the 3-dimensional method of lines for analyzing the electric field within an electrooptic modulator. Although a rather simple electrode shape is presented in the analysis, the program can be used to handle more complicated electrodes with only a little modification.

The validity of the analysis has been verified. The field distribution near the end of the electrode has been illustrated to investigate the end effect. Finally included is the analysis of the capacitance associated with the abrupt stop of the electrodes.

\section{REFERENCES}

[1] P. S. Cross and R. V. Schmidt, "A 1Gbit/s integrated optical modulator," IEEE J. Quantum Electron., vol.15, pp.14151418, Dec. 1979.

[2] R. C. Alferness, S. K. Korotky, and E. A. J. Marcatili, "Velocity-matching techniques for integrated optic traveling wave switch/modulators," IEEE J. Quantum Electron., vol.20, pp.301-309, Mar. 1984.

[3] S. K. Korotcky, etc., "Fully connected high-speed Ti:LiNbO switch/ modulator for time-division multiplexing and data encoding," IEEE J. Lightwave Tech., vol.3, pp.1-6, Feb. 1985.

[4] K. Matsumoto, M. Izutsu, and T. Sueta, "Microwave phase shifter using optical waveguide structure," IEEE J. Lightwave. Tech., vol.9, pp.1523-1527, Nov. 1991.

[5] C. M. Kim and R. V. Ramaswamy, "Overlay integral factors in integrated optic modulators and switches," IEEE J. Lightwave Tech., vol.7, pp.1063-1070, July 1989. 
[6] O. G. Ramer, "Integrated optic electrooptic modulator electrode analysis," IEEE J. Quantum Electron., vol.18, pp.386392, Mar.1982.

[7] J. S. Wei, "Distributed capacitance of planar electrodes in optic and acoustic surface wave devices," IEEE J. Quantum Electron., vol.13, pp.152-158, Apr. 1977.

[8] D. Marcuse, "Optimal electrode design for integrated optics modulators," IEEE J. Quantum Electron., vol.18, pp.393-398, Mar. 1982.

[9] A. G. Keen, M. J. Wale, M. I. Sobhy, and A. J. Holden, "Quasistatic analysis of electrooptic modulators by the method of lines," IEEE J. Lightwave Tech., vol.8, pp.42-50, Jan. 1990.

[10] R. V. Mustacich, "Scalar finite element analysis of electrooptic modulation in diffused channel waveguides and poled waveguides in polymer thin films," Applied Optics, vol.27, pp.3732-
3737, Sept. 1988.

[11] J. C. Yi, S. H. Kim, and S. S. Choi, "Finite-element method for the impedance analysis of traveling-wave modulators," IEEE J. Lightwave Tech., vol.8, pp.817-822, June 1990.

[12] D. Marcuse, "Electrostatic field of coplanar lines computed with the point matching method," IEEE J. Quantum Electron., vol.25, pp.939-947, May 1989.

[13] H. Jin, R. Vahldieck, M. Bélanger, and Z. Jacubczyk, "A mode projecting method for the quasi-static analysis of electrooptic device electrodes considering finite metalization thickness and anisotropic substrate," IEEE J. Quantum Electron., vol.27, pp.2306-2314, Oct.,1991.

[14] R. Pregla and W. Pascher, "The Method of Lines," Ch.6, in Numerical Techniques for Microwave and Millimeter-Wave Passive Structures, ed. by 'T.Itoh, New York: John Wiley, 1989. 